\title{
Cognitive impairment and psychiatric symptoms in normal tension glaucoma (NTG) - A naturalistic study
}

\author{
Mohammad Z Mustafa ${ }^{1 *}$, Tej Rane-Malcolm ${ }^{1}$, Andrew J Tatham ${ }^{1}$, Bal Dhillon ${ }^{1}$, Amanda Cotton ${ }^{2}$, Premal Shah ${ }^{2}$ and Roshini Sanders $^{3}$ \\ ${ }^{1}$ Princess Alexandra Eye Pavilion, Chalmers Street, Edinburgh, United Kingdom \\ ${ }^{2}$ Royal Edinburgh, Department of Psychiatry, Edinburgh, United Kingdom \\ ${ }^{3}$ Queen Margaret Hospital, Department of Ophthalmology, Dunfermline, United Kingdom
}

\begin{abstract}
Purpose: To examine the cognitive and neuropsychiatric characteristics of patients with Normal Tension Glaucoma (NTG).

Methods: A cross-sectional observational study of 99 patients with glaucoma and untreated intraocular pressure consistently <22 mmHg on phasing. All subjects underwent cognitive assessment and psychiatric symptom screening using the mini mental state examination (MMSE), adult verbal learning test (AVLT) and general health questionnaire (GHQ). All subjects had comprehensive ophthalmic examination and documentation of past medical and psychiatric history.

Results: Subjects had a mean age of 70.5 years and $59(59 \%)$ were female. 57 (57\%) had an advanced visual field defect in the worse eye, 19 (19\%) a moderate defect and $23(23 \%)$ a mild defect. There were a high proportion of patients with impaired MMSE, AVLT or GHQ scores. 28 (28\%) had significant short-term memory impairment on AVLT, and 28 (28\%) scored 5 or more on the GHQ suggesting psychiatric caseness. 12 (12\%) scored 24 or less on the MMSE. 43 subjects underwent CT or MRI neuroimaging, with 13 (30\%) showing age excessive generalised cerebral atrophy and 9 (20\%) showing excessive small vessel disease. 41 patients had systemic hypertension, 28 is chaemic heart disease, 21 diabetes, 18 migraine, 13 transient ischaemic attack and 3 Raynaud's disease.

Conclusion: Patients with NTG had a high prevalence of cognitive impairment and psychiatric symptoms identified using common screening tools, suggesting a possible shared intraocular pressure independent mechanism of disease. Ophthalmologists should be aware of the possibility of undiagnosed cognitive impairment and psychiatric morbidity in some patients with NTG.
\end{abstract}

\section{Introduction}

Normal tension glaucoma (NTG) is defined as a form of primary open angle glaucoma (POAG) where intraocular pressure (IOP) is consistently less than $22 \mathrm{mmHg}$. While this definition has limitations, due to population differences in what constitutes a "normal" pressure and diurnal and day-to-day fluctuation in IOP, it is likely that the relative importance of IOP and non-IOP related mechanisms of glaucomatous damage vary between those that develop glaucoma at low and high IOP. Worldwide the prevalence of NTG varies. In people of European ancestry, NTG is thought to affect as many as $50 \%$ of patients suffering with POAG, while in the Japanese population NTG accounts for up to $92 \%$ of glaucoma [1,2].

The diagnosis of NTG can be challenging and, perhaps due to the persistent perception that raised IOP and glaucoma are synonymous, patients may present late. Previous studies have shown a disproportionate number of patients with NTG are registered blind. For example, we previously examined a 20 -year period of blind registrations in Fife, a region of Scotland with a population of 400000, and found those with NTG were over represented in blind registrations $[3,4]$. The management of NTG is also challenging. Although studies such as the Collaborative Normal Tension Glaucoma Study have shown that medical or surgical reduction of IOP reduces the risk of disease progression [5], some patients' progress despite IOP reduction suggesting susceptibility to non-IOP mediated retinal ganglion cell damage [6]. Glaucoma is a neurodegenerative disease characterised by changes to the optic nerve head, however there is growing evidence that the entire visual pathway is affected [7]. This raises the possibility that patients with glaucoma may exhibit non-ocular signs of neurodegeneration. Several studies investigating this theory have suggested an association between glaucoma and neurodegenerative diseases such as dementia $[8,9]$. For example, the recent Three-CityAlienor Cohort study found a high prevalence of glaucoma in patients with dementia, especially NTG [10]. An association between glaucoma and Alzheimer's disease (AD) has also been described, and it has been proposed that both conditions involve neuro-inflammation, decreased cerebrospinal fluid pressure and biomechanical stress and that $\mathrm{B}$-amyloid may have a toxic effect on RGC apoptosis [11-13]. There is also evidence that vascular dysfunction contributes to glaucomatous optic neuropathy, with systemic vascular diseases including stroke and ischemic vascular disease, as well as vasospastic conditions such as Raynaud's disease and migraine, associated with a higher risk of NTG [14-17].

The aim of the current study was to examine the cognitive and

Correspondence to: Mohammad Z Mustafa, Princess Alexandra Eye Pavilion, Chalmers Street, Edinburgh, EH3 9HA, United Kingdom, Tel: 0131 5361000; E-mail: mohammadmustafa@nhs.net

Key words: normal tension glaucoma, psychiatric disease, cognitive profile, visual field defect

Received: February 04, 2016; Accepted: March 12, 2016; Published: March 16, 2016 
neuropsychiatric characteristics of a naturalistic clinical series of patients with NTG using previously validated psychiatric tools. Identifying disease associations may provide further insight into disease mechanisms. Cognitive impairment also has implications for treatment concordance so it is possible that identification of cognitive or psychiatric symptoms could potentially help improve outcomes.

\section{Methods}

This was a cross-sectional observational study including 132 consecutive patients with a diagnosis of NTG attending the ophthalmology department at NHS Fife, Scotland in the year 2009. NTG was defined by the presence of glaucomatous optic neuropathy on slit lamp biomicroscopy identified by a glaucoma specialist (R.S.) and an untreated IOP consistently $<22 \mathrm{mHg}$ using Goldmann applanation tonometry. Ninety-nine of these patients consented to undergo cognitive assessment and psychiatric symptom screening. Of the remaining 33 patients, 8 lacked capacity to consent due to advanced cognitive impairment requiring long-term care. Fifteen were serial non-compliers with appointments and medications and had high fixation errors at visual field testing. Six declined to take part in the study.

Subjects provided written informed consent before participation and the study conformed to the tenets of the Declaration of Helsinki. The Fife ethics committee granted ethical approval prospectively.

All patients with a possible diagnosis of glaucoma referred from community optometrists in Fife, Scotland were streamlined to the NHS specialist glaucoma service comprising higher surgical trainees, specialist glaucoma nurses and hospital optometrists under the supervision of a consultant glaucoma specialist (R.S). At the initial visit all patients had a full ophthalmic examination, including visual acuity, refraction, detailed stereoscopic biomicroscopy of the anterior segment, gonioscopy, IOP measurement with Goldman applanation tonometry, dilated fundus examination, stereoscopic photography of the optic disc and standard automated perimetry (SAP) using the 24-2 Swedish Interactive Threshold Algorithm standard strategy of the Humphrey Field Analyser II (Carl Zeiss Meditec, Dublin, CA, USA). A comprehensive medical history was also taken including history of smoking, migraine, Raynaud's disease, hypertension, ischaemic heart disease, stroke, transient ischaemic attack, peripheral vascular disease and diabetes. Routine haematological indices were evaluated including full blood count, ESR, plasma viscosity, urea and electrolytes, glucose, cholesterol, lipid profile, B12 and folate levels.

Glaucoma was defined by the presence of glaucomatous changes to the optic nerve head or retinal nerve fibre layer on stereoscopic biomicroscopy or stereophotographs. The study only included patients with repeatable glaucomatous defects on SAP and those with open angles on gonioscopy. We excluded patients with secondary glaucoma, including pseudoexfoliation and pigmentary glaucoma. To establish the presence of NTG, patients with glaucomatous changes and an untreated IOP $<22 \mathrm{mmHg}$ at presentation underwent phasing. Phasing consisted of 2 hourly IOP measurements between 8 am to $4 \mathrm{pm}$. Patients with IOP measurements $<22 \mathrm{mmHg}$ at all times points were deemed to have NTG.

Patients with non-glaucomatous optic neuropathies were excluded. We also excluded patients with blood disorders such as polycythaemia and multiple myeloma, those with carotid atherosclerotic disease and those with visual field loss secondary to stroke or other neurological or other non-glaucomatous ocular and non-ocular pathologies.
Neuroimaging including CT or MRI was only performed if there were atypical findings such as significant asymmetric disease or suspicion of neurological disease or a space-occupying lesion.

Glaucomatous visual field defects were classified as mild, moderate or severe using a simplified Hoddapp classification, whereby SAP mean deviation $(\mathrm{MD}) \geq-6 \mathrm{~dB}$ is an early defect; $\mathrm{MD}$ between $6 \mathrm{~dB}$ and $12 \mathrm{~dB}$ is a moderate defect; and MD worse than $-12 \mathrm{~dB}$ is a severe defect. Only reliable visual fields were included and unreliable visual fields were repeated [18].

\section{Cognitive and psychiatric assessment}

All subjects underwent cognitive and psychiatric assessment using three common screening tools - the Mini-mental state examination (MMSE), the General Health Questionnaire (GHQ), and the Auditory verbal learning test (AVLT). All assessments were carried out in one interview by a two trained psychiatrists (AC and SDH). This was done over 18 months between 2010 and 2012. In addition patients were also asked whether they had any history of having a 'problem with their nerves', had ever consulted a psychiatrist or psychologist, had been on medication for their 'nerves', or whether they had any current involvement with mental health services.

The mini mental state examination (MMSE) is an established widely used screening tool for identifying cognitive impairment [19]. It comprises sections relating to orientation, registration, attention, calculation and language, with a full score of 30 . Mild cognitive impairment is defined as a score between $21-24$, moderate between 10-20 and severe less than 9 [19]. The test is commonly used in clinical practice to identify those with significant cognitive impairment in adult groups including the elderly.

The General Health Questionnaire (GHQ- 28) is a screening tool for psychiatric symptoms [20]. The GHQ has 4 sub-scales, each with 7 items. The subscales include somatic symptoms, anxiety and insomnia, social dysfunction and severe depression. We used the recommended method for identifying 'caseness' [21], whereby each item is scored between 0 and 1. A score of 5 or more is the accepted threshold for identifying patients with a high probability of a psychiatric diagnosis across all age groups including the elderly.

The AVLT is a well-established and validated measure of verbal learning and memory [22]. A list of 15 unrelated concrete nouns (List A) is read aloud in the same order on five consecutive occasions. After each reading, participants recall as many of the words as possible in any order. After the fifth recall session, participants are read a distractor list (List B) of 15 different concrete nouns, followed by a free-recall test from List B. Immediately afterwards, participants are asked to free recall words from List A. After a 20-minute interval, participants are again asked to recall words from List A (delayed recall). Participant's performance at each stage was standardised into z-scores (i.e. the results were expressed as the number of standard deviations above or below the appropriate established age and gender adjusted mean) [2224].

\section{Statistical analysis}

All statistical analysis was conducted using IBM SPSS Statistics for Macintosh (Version 22.0. Armonk, NY: IBM Corp, Released 2013).

\section{Results}

The 99 subjects included in the study had a mean ( \pm 12 years) age of $70.5 \pm 0.5$ years. The demographic and clinical characteristics of 
patients included in the study are shown in Table 1. Fifty-nine (59\%) of participants were female (Table 1). One hundred and sixty eight eyes (85\%) had vision better than $6 / 12$ at presentation. Twenty-three (23\%) had mild glaucomatous visual field loss in the worse eye, 19 (19\%) moderate loss and 57 (57\%) severe visual field loss. There were no significant differences in gender $(\mathrm{p}<0.73)$ and age $(\mathrm{p}<0.38)$ between the 3 groups of glaucoma severity.

Comorbidities were common. Forty-one (41\%) subjects had systemic hypertension, 28 (28\%) ischaemic heart disease, $21(21 \%)$ diabetes, $13(13 \%)$ had a history of transient ischaemic attacks, and $7(7 \%)$ peripheral vascular disease. 18 (18\%) suffered from migraine and 3 (3\%) had Raynaud's Syndrome. Thirty-one patients were current smokers.

There were a high proportion of patients with impaired MMSE, AVLT or GHQ scores. Twelve patients (12\%) had abnormal MMSEs, 7 with mild, 3 moderate and 2 severe cognitive deficits (Table 2). Ten of the 12 patients $(83 \%)$ with abnormal MMSE had advanced visual field defects and of 57 patients with advanced field defects, 10 (18\%) had abnormal MMSEs.

On the GHQ, 28 patients (28\%) scored 5 or more, suggesting a high probability of having a psychiatric diagnosis. The highest scores tended to be in relation to somatic symptoms, followed by anxiety and insomnia then social dysfunction and finally severe depression. On the AVLT, 28\% of patients were 2 standard deviations (SD) or less than expected for age and gender adjusted means in terms of short-term verbal memory performance (Figure 1), 25\% were 2 SD or less for verbal learning (Figure 2) and 22\% were 2 SDs or less for long-term verbal memory (Figure 3 ). Normally, only $2.5 \%$ of the sample would be expected to score $2 \mathrm{SD}$ or less than the age and IQ adjusted population mean.

Twenty one patients (21\%) admitted to having had 'problem with their nerves' at some stage, $11(11 \%)$ were currently on medication for their 'nerves', 9 (9\%) had previously seen a psychiatrist, but only 3

Table 1. Characteristics of the study group.

\begin{tabular}{|l|l|}
\hline Demographic Characteristics & $\mathrm{N}=99$ \\
\hline Age, years mean (SD) & $70.5(12.0)$ \\
\hline Gender & 59 female $(59.5 \%)$ \\
\hline Visual Fields & $23(23.2)$ \\
\hline Mild & $19(19.2)$ \\
\hline Moderate & $57(57.6)$ \\
\hline Severe & \\
\hline Medical Characteristics & \\
\hline Diabetes & $21(21 \%)$ \\
\hline Hypertension & $41(41.4)$ \\
\hline Smoking & $31(31.3)$ \\
\hline TIA & $13(13.1)$ \\
\hline Ischaemic Heart Disease & $28(28.3)$ \\
\hline Raynauds & $3(3.0)$ \\
\hline Migraine & $18(18.2)$ \\
\hline Peripheral Vascular Disease & \\
\hline Psychiatric Screening Questions & $7(7.1)$ \\
\hline Problems with "nerves' & \\
\hline Ever seen a Psychiatrist & $21(21.2 \%)$ \\
\hline On medication for 'nerves' & $9(9.1 \%)$ \\
\hline Currently involved with Mental Health Services & $11(11.1 \%)$ \\
\hline & $3(3.03 \%)$ \\
\hline & \\
\hline
\end{tabular}

Table 2. MMSE and GHQ results

\begin{tabular}{|l|c|}
\hline MMSE, No. (\%) & Total $\mathbf{n = 9 9}$ \\
\hline Normal & $87(87.9)$ \\
\hline Mild & $7(7.0)$ \\
\hline Moderate & $3(3.0)$ \\
\hline Severe & $2(2.0)$ \\
\hline Mean MMSE & 27.5 \\
\hline GHQ & \\
\hline Caseness, No. (\%) & $28(28.3)$ \\
\hline Mean (GHQ Method) & 4.01 \\
\hline
\end{tabular}

(3\%) were currently involved with mental health services. Forty-three (43\%) patients underwent neurological imaging. Thirteen patients had evidence of generalised cerebral atrophy and nine patients had micro vascular disease in excess of that expected for age. This data was reported and sourced from the specialist neuroradiology reports provided by our regional service.

\section{Discussion}

The results from this study suggest a high prevalence of cognitive impairment and psychiatric symptomatology in patients with NTG. In our cohort, $12 \%$ of patients had cognitive impairment identified on the MMSE, which is higher than one would expect for healthy subjects of a similar age. A recent meta-analysis indicated a global prevalence of dementia in relation to cognitive impairment of 5\%-7\% [25], in those aged 60 years or over. Our study suggests that the prevalence of cognitive impairment in patients with NTG may be twice that.

The GHQ showed that more than one in four (28\%) of those with NTG had a high probability of having a psychiatric diagnosis. Furthermore, over one quarter of patients had AVLT scores 2 SD or less than the expected age matched population mean. The Rey AVLT test is a sensitive tool that measures a number of key aspects of memory, including short-term verbal memory, verbal learning and long-term verbal memory abilities. The results therefore suggest that there is a higher prevalence of short-term memory impairment in patients' with NTG compared to an age matched reference population. [22-24] Moreover as some patients were excluded from the study due to advanced psychiatric illness, this further suggests a high prevalence of psychiatric and cognitive problems in those with NTG.

To our knowledge this is the first study to systematically use validated screening tools to quantify cognitive impairment and psychiatric symptomatology in patients with NTG. Previous studies have however reported a higher prevalence of dementia, cognitive impairment and depression in patients with glaucoma [26,27], and a high prevalence of glaucoma in those with Alzheimer's disease $[13,28,29]$. Yochin et al. also reported correlation between depression and increasing glaucoma severity [27]. In the present study we found patients with NTG were more likely to report somatic rather than depressive symptoms using the GHQ. The high prevalence of psychiatric symptoms may also be a result of the impact of glaucoma and glaucoma treatment on quality of life. However, despite the high prevalence of cognitive impairment and psychiatric symptoms, only 3 patients were currently in contact with mental health services. This suggests that ophthalmologists should consider whether some patients with NTG might benefit from formal assessment by mental health services.

Almost $57 \%$ of patients included in this study had an advanced 


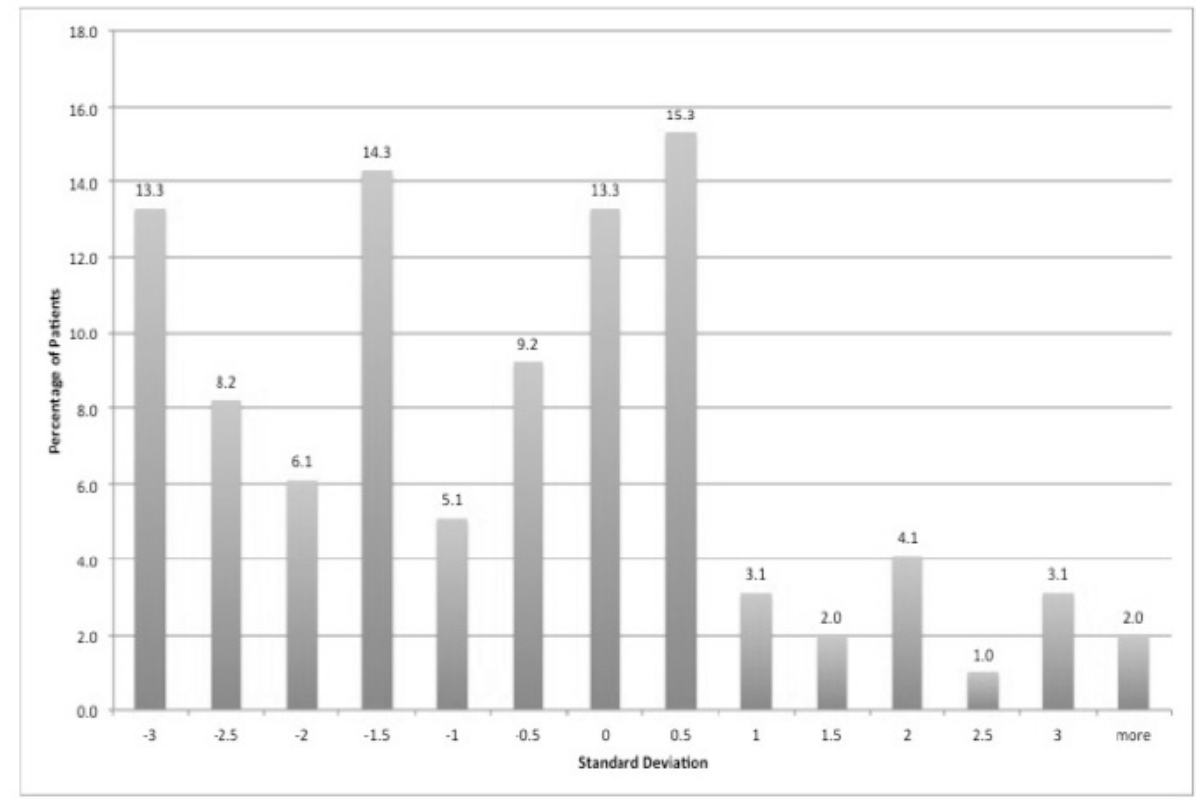

Figure 1. Short Term Verbal Memory AVLT. Showing 28\% of patients were 2 SD or less than expected for age and gender adjusted means.

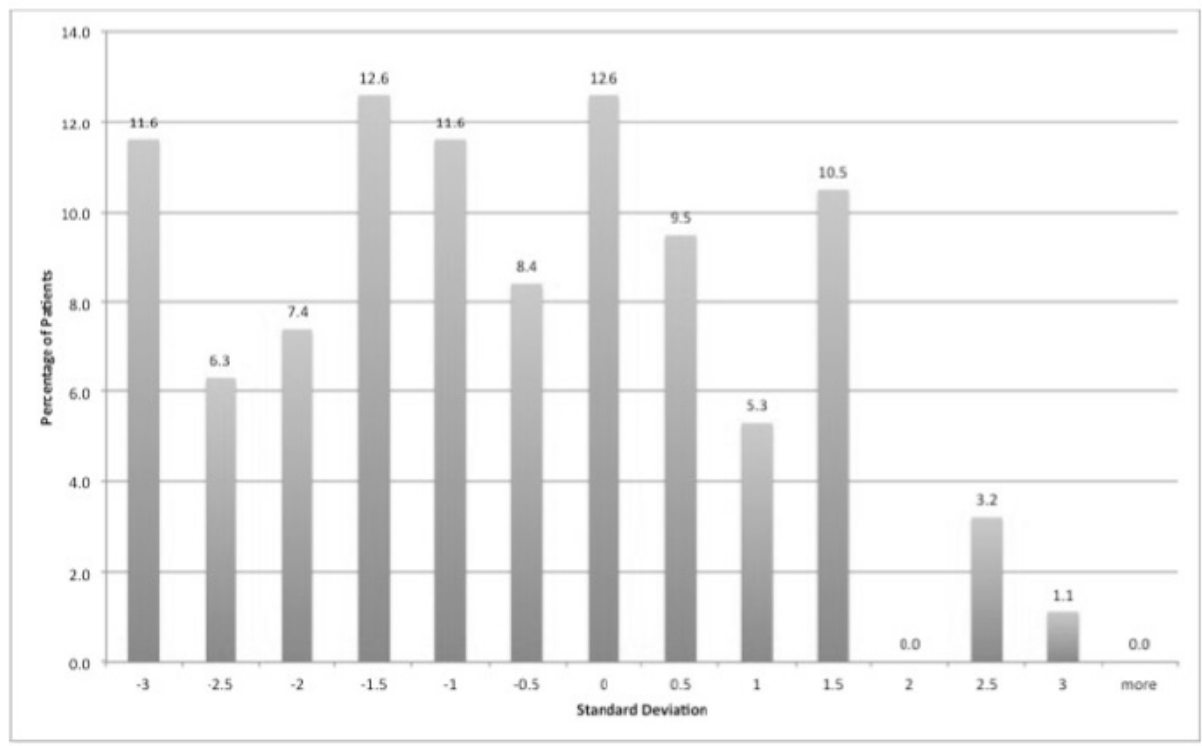

Figure 2. Verbal learning AVLT. Showing $25 \%$ of patients were 2 SD or less than expected for age and gender adjusted means.

visual field defect in at least on eye at presentation, which is in agreement with previous studies showing patients with NTG are more likely to present with advanced disease [30]. It has also been shown that factors including older age, African ancestry and socioeconomic deprivation are associated with delayed presentation [31-35].

Although some studies have examined the association between glaucoma and dementia [8, 10-13], few have specifically examined psychiatric and cognitive associations. Yochim BP et al. found $20 \%$ of patients with POAG had memory impairment, which is comparable to the $28 \%$ found in similarly aged patients with NTG in our study [27]. They also found, from the same sample of patients $22 \%$ had impaired executive functioning using the California Verbal Learning Test and just over $12 \%$ of patients had mild to moderate depressive symptoms [27]. The GHQ suggested that $28 \%$ of our sample had a high probability of having a psychiatric disorder, thus potentially requiring neuropsychiatric assessment. As only $3 \%$ of the patients currently had contact with mental health services, it may be advisable for patients presenting with NTG to be routinely screened for mental health problems. There may also be implications on compliance of treatment exacerbated by diminished cognition, memory impairment and possibly poor mental health. Certainly, studies have shown a poor adherence to medications related to psychosocial factors [36].

This study has several limitations. First, due to the limitations of current diagnostic tools, the use of the term NTG is controversial and may not be a distinct disease from POAG. IOP is known to fluctuate and in many patients is highest outside office hours. Although we conducted office hour phasing of IOP for all subjects, it is quite possible that patients had IOP above $21 \mathrm{mmHg}$ at other times or on days when 


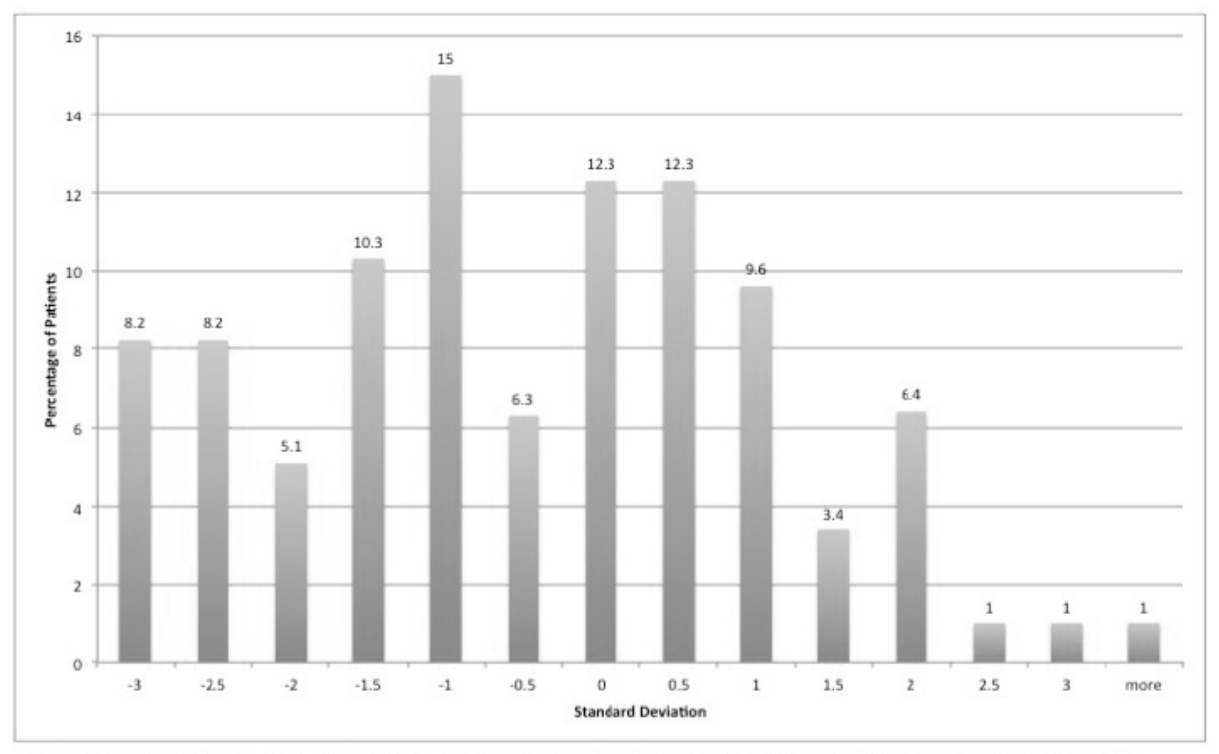

Figure 3. Delayed Recall AVLT. Showing $22 \%$ of patients were 2 SDs or less than expected for age and gender adjusted means.

they were not attending the hospital for examination. Furthermore, in order to recruit sufficient numbers of patients, the study was commenced in 2009 , prior to pachymetry being routinely available in our unit. Unfortunately for this reason corneal thickness data was not available for all patients. However, even if corneal thickness was known, one could argue that as IOP measurements are also affected by factors such as corneal hysteresis, the true IOP remains uncertain [37]. We therefore chose to adopt a pragmatic approach, with phasing of IOP representing current clinical practice. However, the cut off value of $21 \mathrm{mmHg}$ for NTG, which is based on population data for normal IOP ranges, is not applicable for all populations and it is hard to conceive that patients with glaucoma and untreated IOPs of $20 \mathrm{mmHg}$ or $22 \mathrm{mmHg}$ have different diseases. It is however useful to phenotype patients and those with consistently low IOPs may have different factors contributing to glaucomatous damage, compared to those with high pressures. If further studies corroborate an association between cognitive and psychiatric difficulties and NTG, this would support the concept of a possible shared non-IOP dependent neurodegenerative pathological process. Future studies should also include patients with POAG with higher IOPs, to determine whether the high prevalence of cognitive deficit and abnormal mental health is also present in these patients.

The GHQ and MMSE are not diagnostic tests but are screening instruments for general mental health symptoms and for general cognitive impairment. The AVLT is a more specific and sensitive instrument which measures aspects of verbal memory but is not diagnosis specific. Not withstanding this, our study suggests a higher frequency of cognitive deficit and mental health symptoms amongst patients with NTG. These may significantly impact on treatment compliance, and glaucoma-associated visual loss potentially may compound the disability associated with cognitive impairment. If neuropsychiatric morbidities remain undetected it is likely to have a negative impact on ocular and systemic health and wellbeing. Few of the patients in our study were in contact with mental health services and it is likely this is reflective of a wider unmet need in health service provision in patients attending glaucoma clinics in other UK regions. We suggest that further research is needed to explore associations between glaucomatous optic neuropathy, cognitive decline and psychiatric co-morbidities in order to better understand the relevance of shared pathogenesis and improve patient care.

\section{Summary}

What was known before:

- Glaucoma is a neurodegenerative disease.

- Glaucoma is associated with other neurodegenerative diseases such as Alzheimer's disease.

What this study adds:

- Quantifies psychiatric and cognitive profile of patients with normal tension glaucoma.

- Suggests that NTG patients may need to be routinely screened for neuropsychiatric symptoms with further psychiatric assessment thereafter.

- Cognitive and neuropsychiatric problems could impact on a patient's compliance with NTG treatment.

- $\quad$ Ophthalmic treatment may have to be modified taking into account these neuropsychiatric findings.

\section{Contributorship statement}

Mohammad Z Mustafa (MM) wrote the article with editing from Andrew J Tatham (AT), Bal Dhillon (BD) and Roshini Sanders (RS). The study was designed by RS and BD with glaucoma expertise from AT. Tej Rane-Malcolm carried out data collection with cognitive testing carried out by Premal Shah (PS) and Amanda Cotton. Data analysis was carried out by MM and PS. RS was project lead and also carried out all clinical ophthalmic examination.

\section{Funding}

There was no funding required for this study. 


\section{Competing interests}

Andrew J Tatham receives research support from Heidelberg Engineering and Thea.

\section{References}

1. Sommer A, Tielsch JM, Katz J, Quigley HA, Gottsch JD, et al (1991) Relationship between intraocular pressure and primary open angle glaucoma among White and Black Americans. Arch Ophthalmol 109: 1090-1095. [Crossref]

2. Iwase A, Suzuki Y, Araie M, Yamamoto T, Abe H, et al. (2004) The prevalence of primary open-angle glaucoma in Japanese: the Tajimi Study. Ophthalmology 111: 1641-1648. [Crossref]

3. Sinclair A, Hinds A, Sanders R (2004) Ten years of glaucoma blindness in Fife 199099 and the implications for ophthalmology, optometry and rehabilitation services. Ophthalmic Physiol Opt 24: 313-318. [Crossref]

4. O'Colmain U, Anijeet D, Vosoughi M, Sinclair A, Sanders R (2011) Glaucoma blind registration in Fife (2000-2009) - a retrospective cohort study. Ophthalmic Physiol Opt 31: 360-366. [Crossref]

5. [No authors listed] (1998) The effectiveness of intraocular pressure reduction in the treatment of normal-tension glaucoma. Collaborative Normal-Tension Glaucoma Study Group. Am J Ophthalmol 126: 498-505. [Crossref]

6. Mi XS, Yuan TF, So KF (2014) The current research status of normal tension glaucoma. Clin Interv Aging 9: 1563-1571. [Crossref]

7. Gupta N, Yücel YH (2007) Glaucoma as a neurodegenerative disease. Curr Opin Ophthalmol 18: 110-114. [Crossref]

8. McKinnon S (2012) The Cell and Molecular Biology of Glaucoma: Common Neurodegenerative Pathways and Relevance to Glaucoma. Invest Ophthalmol Vis Sci 53: 2485. [Crossref]

9. Stasi K, Nagel D, Yang X, Wang R, Ren L, et al. (2006) Complement Component 1Q (C1Q) Upregulation in Retina of Murine, Primate, and Human Glaucomatous Eyes. Invest Ophthalmol Vis Sci 47: 1024-1029. [Crossref]

10. Helmer C, Malet F, Rougier MB, Schweitzer C, Colin J, et al. (2013) Is there a link between open-angle glaucoma and dementia? The Three-City-Alienor cohort. Ann Neurol 74: 171-179. [Crossref]

11. Tsilis AG, Tsilidis KK, Pelidou SH, Kitsos G (2014) Systematic review of the association between Alzheimer's disease and chronic glaucoma. Clin Ophthalmol 8: 2095-2104. [Crossref]

12. Wostyn P, Audenaert K, De Deyn PP (2009) More advanced Alzheimer's disease may be associated with a decrease in cerebrospinal fluid pressure. Cerebrospinal Fluid Res 6: 14. [Crossref]

13. McKinnon SJ (2003) Glaucoma: ocular Alzheimer's disease? Front Biosci 8: s11401156. [Crossref]

14. Kim C, Kim TW (2009) Comparison of risk factors for bilateral and unilateral eye involvement in normal-tension glaucoma. Invest Ophthalmol Vis Sci 50: 1215-1220. [Crossref]

15. Meyer JH, Brandi-Dohrn J, Funk J (1996) Twenty four hour blood pressure monitoring in normal tension glaucoma. Br J Ophthalmol 80: 864-867. [Crossref]

16. Hulsman CA, Vingerling JR, Hofman A, Witteman JC, de Jong PT (2007) Blood pressure, arterial stiffness, and open-angle glaucoma: the Rotterdam study. Arch Ophthalmol 125: 805-812. [Crossref]

17. Furlanetto RL, De Moraes CG, Teng CC, Liebmann JM, Greenfield DS, et al. (2014) Risk factors for optic disc hemorrhage in the low-pressure glaucoma treatment study. Am J Ophthalmol 157: 945-952. [Crossref]
18. Hodapp E, Parrish R, Anderson D (1993) Clinical decisions in glaucoma. St. Louis, Mo.: Mosby, pp.52-61.

19. Tombaugh TN, McIntyre NJ (1992) The mini-mental state examination: a comprehensive review. J Am Geriatr Soc 40: 922-935. [Crossref]

20. Goldberg DP, Blackwell B (1970) Psychiatric illness in general practice. A detailed study using a new method of case identification. $\operatorname{Br}$ Med $J$ 1: 439-443. [Crossref]

21. Dale B, Söderhamn U, Söderhamn O (2012) Psychometric properties of the Norwegian version of the General Health Questionnaire (GHQ-30) among older people living at home. Psychol Res Behav Manag 5: 151-157. [Crossref]

22. Messinis L, Tsakona I, Malefaki S, Papathanasopoulos P (2007) Normative data and discriminant validity of Rey's Verbal Learning Test for the Greek adult population. Arch Clin Neuropsychol 22: 739-752. [Crossref]

23. Balzano J, Chiaravalloti N, Lengenfelder J, Moore N, DeLuca J (2006) Does the scoring of late responses affect the outcome of the paced auditory serial addition task (PASAT)? Arch Clin Neuropsychol 21: 819-825. [Crossref]

24. Rosenberg SJ, Ryan JJ, Prifitera A (1984) Rey Auditory-Verbal Learning Test performance of patients with and without memory impairment. J Clin Psychol 40: 785787. [Crossref]

25. Prince M, Bryce R, Albanese E, Wimo A, Ribeiro W, et al. (2013) The global prevalence of dementia: a systematic review and metaanalysis. Alzheimers Dement 9: 63-75. [Crossref]

26. Skalicky S, Goldberg I (2008) Depression and quality of life in patients with glaucoma: A cross-sectional analysis using the geriatric depression scale-15, assessment of function related to vision, and the glaucoma quality of life-15. J Glaucoma 17: 546551. [Crossref]

27. Yochim BP, Mueller AE, Kane KD, Kahook MY (2012) Prevalence of cognitive impairment, depression, and anxiety symptoms among older adults with glaucoma. $J$ Glaucoma 21: 250-254. [Crossref]

28. Bayer AU, Ferrari F, Erb C (2002) High occurrence rate of glaucoma among patients with Alzheimer's disease. Eur Neurol 47: 165-168. [Crossref]

29. Tamura H, Kawakami H, Kanamoto T, Kato T, Yokoyama T, et al. (2006) High frequency of open-angle glaucoma in Japanese patients with Alzheimer's disease. $J$ Neurol Sci 246: 79-83. [Crossref]

30. Wallace E, Paterson H, Miller S, Sinclair A, Sanders R et al. (2008) Patient profile and management in advanced glaucoma. Br J Vis Impairm 26: 7-23.

31. Grant WM, Burke JF Jr (1982) Why do some people go blind from glaucoma? Ophthalmology 89: 991-998. [Crossref]

32. Parc CE, Johnson DH, Oliver JE, Hattenhauer MG, Hodge DO (2001) The long-term outcome of glaucoma filtration surgery. Am J Ophthalmol 132: 27-35. [Crossref]

33. Ng WS, Agarwal PK, Sidiki S, McKay L, Townend J, et al. (2010) The effect of socioeconomic deprivation on severity of glaucoma at presentation. Br J Ophthalmol 94 85-87. [Crossref]

34. Fraser S, Bunce C, Wormald R, Brunner E (2001) Deprivation and late presentation of glaucoma: case-control study. BMJ 322: 639-643. [Crossref]

35. Sukumar S, Spencer F, Fenerty C, Harper R, Henson D (2009) The influence of socioeconomic and clinical factors upon the presenting visual field status of patients with glaucoma. Eye (Lond) 23: 1038-1044. [Crossref]

36. Lim MC, Watnik MR, Imson KR, Porter SM, Granier AM (2013) Adherence to glaucoma medication: the effect of interventions and association with personality type. J Glaucoma 22: 439-446. [Crossref]

37. Ventura AC, Böhnke M, Mojon DS (2001) Central corneal thickness measurements in patients with normal tension glaucoma, primary open angle glaucoma, pseudoexfoliation glaucoma, or ocular hypertension. Br J Ophthalmol 85: 792-795. [Crossref]

Copyright: (C2016 Mustafa MZ. This is an open-access article distributed under the terms of the Creative Commons Attribution License, which permits unrestricted use, distribution, and reproduction in any medium, provided the original author and source are credited. 